\title{
Mott Insulators in the Strong Spin-Orbit Coupling Limit: From Heisenberg to a Quantum Compass and Kitaev Models
}

\author{
G. Jackeli ${ }^{1, *}$ and G. Khaliullin ${ }^{1}$ \\ ${ }^{1}$ Max-Planck-Institut für Festkörperforschung, Heisenbergstrasse 1, D-70569 Stuttgart, Germany
}

(Dated: December 4, 2008)

\begin{abstract}
We study the magnetic interactions in Mott-Hubbard systems with partially filled $t_{2 g}$-levels and with strong spin-orbit coupling. The latter entangles the spin and orbital spaces, and leads to a rich variety of the low energy Hamiltonians that extrapolate from the Heisenberg to a quantum compass model depending on the lattice geometry. This gives way to "engineer" in such Mott insulators an exactly solvable spin model by Kitaev relevant for quantum computation. We, finally, explain "weak" ferromagnetism, with an anomalously large ferromagnetic moment, in $\mathrm{Sr}_{2} \mathrm{IrO}_{4}$.

PACS numbers: $75.30 . \mathrm{Et}, 71.70 . \mathrm{Ej}, 75.10 . \mathrm{Jm}$
\end{abstract}

The transition metal compounds with partially filled $d$-levels have been the subject of extensive studies after the discovery of a variety of novel physical phenomena and a diversity of new phases $[1,2,[3]$. In the undoped compounds a strong Coulomb repulsion localizes the $d$ electrons in Mott-Hubbard or charge-transfer insulating regimes [4]. The low energy physics of such insulators, in some cases, are described in terms of spin-only Hamiltonians. This happens when the symmetry of the local surrounding of a transition metal (TM) ion is low enough to lift the orbital degeneracy of a $d$-level as in case of, e.g., high- $T_{c}$ cuprates. However, often, a TM ion possesses an orbital degeneracy in addition to that originating from spin. Typically, the orbitals form a long-range-ordered pattern driven by Jahn-Teller or exchange interactions, and, being subject to a discrete symmetry, behave more like static (classical) objects compared to their spin partners. Magnetic properties of orbitally ordered systems depend on the symmetry of the occupied orbitals. Indeed, orbital ordering may stabilize various types of magnetic phases [5], as well as spin gapped states without any long-range spin order [6, 7]. In other circumstances, the situation can be opposite: the orbitals may remain in a liquid state down to the lowest temperatures (due to strong quantum fluctuations), while the spins are slowly fluctuating about a long range ordered state [8, 9].

In this Letter we want to discuss yet another situation, when a strong relativistic spin-orbit (SO) coupling entangles locally the spin and orbital degrees of freedom. The physics of such systems may drastically differ from that of compounds in which spin-orbit coupling is of a perturbative nature, as the form of magnetic interactions is no longer dictated by a global spin $S U(2)$ symmetry alone. The effects of a strong SO interaction on cooperative magnetic phenomena has been discussed in the pioneering works by Kanamori on $\mathrm{Fe}^{2+}$ and $\mathrm{Co}^{2+}$ compounds [10]. In recent years, there has been a revival of interest in spin-orbit coupling in the context of exchange interactions [11, 12, 13], magnetoelectric 14] and spin Hall effects [15], Fermi-surface topology [16], etc.
The SO coupling is strongly enhanced for the late transition metal ions such as Ir, Os, Rh, Ru. Indeed, optical absorption data on $\mathrm{Ir}^{4+}$ impurities in $\mathrm{SrTiO}_{3}$ suggests a fairly high value of the SO coupling $\lambda \sim 380 \mathrm{meV}$ [17]. This far exceeds possible intersite interactions between the $t_{2 g}$ orbitals and spins in the insulating iridates, and hence is able to lock them together forming a total angular momentum locally. In the following we focus on the systems composed of magnetic ions with a single hole in a threefold degenerate $t_{2 g}$-level, a low spin state of $d^{5}$-configuration, such as $\mathrm{Ir}^{4+}$ or $\mathrm{Rh}^{4+}$ ions subject to a strong octahedral field. We formulate a superexchange theory for such systems and show that together with conventional interactions of predominantly Heisenberg form, more exotic spin models such as the quantum compass model naturally appear as low energy Hamiltonians. We suggest how to implement in such Mott insulators an exactly solvable model proposed by Kitaev [18], which exhibit exotic anyonic excitations with fractional statistics. We apply the present theory to the insulating iridium compound $\mathrm{Sr}_{2} \mathrm{IrO}_{4}$ [19, 20, 21, 22] exhibiting "weak" ferromagnetism (FM) with an anomalously large FM moment.

Single ion Kramers doublet.- We first introduce the local magnetic degrees of freedom. In the low spin $d^{5}$ configuration a hole resides in $t_{2 g}$ manifold of $x y, x z, y z$ orbitals, and has an effective angular momentum $l=1$ [23]: $\left|l_{z}=0\right\rangle \equiv|x y\rangle,\left|l_{z}= \pm 1\right\rangle \equiv-\frac{1}{\sqrt{2}}(i|x z\rangle \pm|y z\rangle)$. The total moment $\vec{M}=2 \vec{s}-\vec{l}$, where $\vec{s}$ is a hole spin operator. The single ion Hamiltonian $H_{0}=\lambda \vec{l} \cdot \vec{s}+\Delta l_{z}^{2}$ consists of a SO coupling with $\lambda>0$ and a possible tetragonal splitting $\Delta$ of the $t_{2 g}$ levels. $\Delta>0$ for an oxygen octahedron elongated along the $z \| c$-axis. The lowest energy level of $H_{0}$ is a Kramers doublet of isospin states $|\tilde{\uparrow}\rangle$ and $|\tilde{\downarrow}\rangle$ :

$$
\begin{aligned}
& |\tilde{\uparrow}\rangle=\sin \theta|0, \uparrow\rangle-\cos \theta|+1, \downarrow\rangle, \\
& |\tilde{\downarrow}\rangle=\sin \theta|0, \downarrow\rangle-\cos \theta|-1, \uparrow\rangle .
\end{aligned}
$$

Angle $\theta$ parameterizes the relative strength of the tetragonal splitting, with $\tan (2 \theta)=2 \sqrt{2} \lambda /(\lambda-2 \Delta)$. Notice that the wave functions of the Kramers doublet are given 


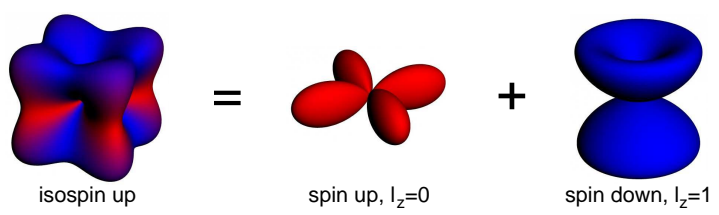

FIG. 1: (Color online) Density profile of a hole in the isospin up state (without tetragonal distortion). It is a superposition of a spin up hole density in $|x y\rangle$-orbital, $l_{z}=0$, (middle) and spin down one in $(|y z\rangle+i|x z\rangle)$ state, $l_{z}=1$, (right).

by a coherent superposition of different orbital and spin states, leading to a peculiar distribution of spin densities in real space (see Fig. 11). This will have important consequences for the symmetry of the intersite interactions. Namely, the very form of the exchange Hamiltonian depends on bond geometry through a density profile of Kramers states, as we demonstrate below.

Exchange couplings of neighboring Kramers states.We consider the limit of the strong spin-orbit coupling, i.e., when $\lambda$ is larger than exchange interaction between the isospins. The exchange Hamiltonians for isospins are then obtained by projecting the corresponding superexchange spin-orbital models onto the isospin states Eq. (1). First, we present the results for the case of cubic symmetry $(\Delta=0, \sin \theta=1 / \sqrt{3})$, and discuss later the effects of a tetragonal distortion. We consider two common cases of TM-O-TM bond geometries: (A) $180^{\circ}$-bond formed by corner-shared octahedra as in Fig. 2(a), and (B) $90^{\circ}$-bond formed by edge-shared ones, Fig. 2(b).

(A) $180^{\circ}$-bond: For this geometry, the nearestneighbor $t_{2 g}$ hopping matrix is diagonal in the orbital space and, on a given bond, only two orbitals are active, e.g., $|x y\rangle$ and $|x z\rangle$ orbitals along a bond in $x$-direction [Fig. 1(a)]. The spin-orbital exchange Hamiltonian for such a system has already been reported: see Eq. (3.11) in Ref. [12]. After projecting it onto the ground state doublet, we find an exchange Hamiltonian for isospins in a form of Heisenberg plus a pseudo-dipolar interaction:

$$
\mathcal{H}_{i j}=J_{1} \vec{S}_{i} \cdot \vec{S}_{j}+J_{2}\left(\vec{S}_{i} \cdot \vec{r}_{i j}\right)\left(\vec{r}_{i j} \cdot \vec{S}_{j}\right)
$$

where $\vec{S}_{i}$ is the $S=1 / 2$ operator for isospins (referred to as simply spins from now on), $\vec{r}_{i j}$ is the unit vector along the $i j$ bond, and $J_{1(2)}=\frac{4}{9} \nu_{1(2)}$. Hereafter, we use the energy scale $4 t^{2} / U$ where $t$ is a $d d$-transfer integral through an intermediate oxygen, and $U$ stands for the Coulomb repulsion on the same orbitals. The parameters $\nu_{1(2)}$ controlling isotropic (anisotropic) couplings are given by $\nu_{1}=\left(3 r_{1}+r_{2}+2 r_{3}\right) / 6$ and $\nu_{2}=\left(r_{1}-r_{2}\right) / 4$, where the set of $r_{n}$ characterizing the multiplet structure of the excited states depends solely on the ratio $\eta=J_{H} / U$ of Hund's coupling and $U$ [24]. At small $\eta$, one has $\nu_{1} \simeq 1$ and $\nu_{2} \simeq \eta / 2$. Thus, we find a predominantly isotropic Hamiltonian, with a weak dipolar-like anisotropy term. While the overall form of Eq. (2) could be anticipated

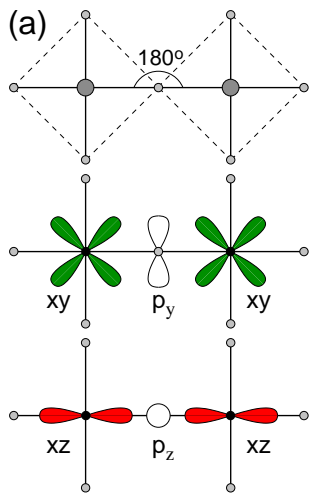

(b)
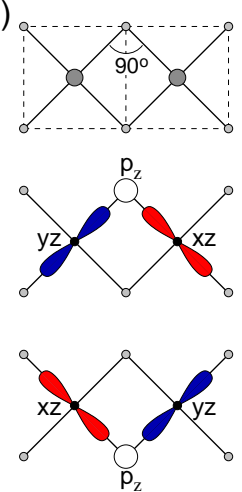

FIG. 2: (Color online) Two possible geometries of a TMO-TM bond with corresponding orbitals active along these bonds. The large (small) dots stand for the transition metal (oxygen) ions. (a) $180^{\circ}$-bond formed by corner shared octahedra, and (b) $90^{\circ}$-bond formed by edge shared octahedra.

from symmetry arguments, the explicit derivation led us to an unexpected result: In the limit of strong SO coupling, the magnetic degrees are governed by a nearly Heisenberg model just like in the case of small $\lambda$, and, surprisingly enough, its anisotropy is entirely due to the Hund's coupling. This is opposite to a conventional situation: typically, the anisotropy corrections are obtained in powers of $\lambda$ while the Hund's coupling is not essential.

(B) $90^{\circ}$-bond: There are again only two orbitals active on a given bond, e.g., $|x z\rangle$ and $|y z\rangle$ orbitals along a bond in the $x y$-plane. However, the hopping matrix has now only non-diagonal elements and there are two possible paths for a charge transfer [via upper or lower oxygen, see Fig. 2(b)]. This peculiarity of a $90^{\circ}$-bond leads to an exchange Hamiltonian drastically different from that of a $180^{\circ}$ geometry. Two transfer amplitudes via upper and lower oxygen interfere in a destructive manner and the isotropic part of the Hamiltonian exactly vanishes. The finite, anisotropic interaction appears, however, due to the $J_{H}$-multiplet structure of the excited levels. Most importantly, the very form of the exchange interaction depends on the spatial orientation of a given bond. We label a bond $i j$ laying in the $\alpha \beta$ plane perpendicular to the $\gamma(=x, y, z)$ axis by a $(\gamma)$-bond. With this in mind, the Hamiltonian can be written as:

$$
\mathcal{H}_{i j}^{(\gamma)}=-J S_{i}^{\gamma} S_{j}^{\gamma}
$$

with $J=\frac{4}{3} \nu_{2}$. Remarkably, this Hamiltonian is precisely a quantum analog of the so-called compass model. The latter, introduced originally for the orbital degrees of freedom in Jahn-Teller systems [5], has been the subject of numerous studies as a prototype model with protected ground state degeneracy of topological origin (see, e.g., Ref. 25). However, to our knowledge, no magnetic realization of the compass model has been proposed so far.

Implementing the Kitaev model in Mott insulators.The Kitaev model is equivalent to a quantum compass 


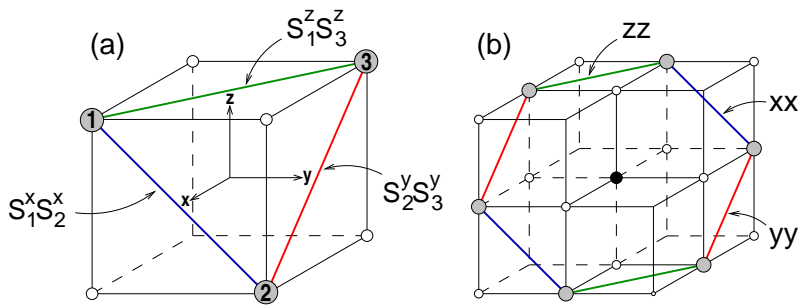

FIG. 3: (Color online) Examples of the structural units formed by $90^{\circ}$ TM-O-TM bonds and corresponding spincoupling patterns. Grey circles stand for magnetic ions, and small open circles denote oxygen sites. (a) Triangular unit cell of $\mathrm{ABO}_{2}$-type layered compounds, periodic sequence of this unit forms a triangular lattice of magnetic ions. The model (3) on this structure is a realization of a quantum compass model on a triangular lattice: e.g., on a bond 1-2, laying perpendicular to $x$-axis, the interaction is $S_{1}^{x} S_{2}^{x}$. (b) Hexagonal unit cell of $\mathrm{A}_{2} \mathrm{BO}_{3}$-type layered compound, in which magnetic ions (B-sites) form a honeycomb lattice. (Black dot: nonmagnetic A-site). On an $x x$-bond the interaction is $S_{i}^{x} S_{j}^{x}$, etc. For this structure the model (3) is identical to the Kitaev model.

model on a honeycomb lattice [26]. It shows a number of fascinating properties such as anyonic excitations with exotic fractional statistics, topological degeneracy, and, in particular, it is relevant for quantum computation [18]. This generated an enormous interest in a possible realization of this model in real systems, with current proposals based on optical lattices [27]. Here we outline how to "engineer" the Kitaev model in Mott insulators.

Shown in Fig. 3(a) is a triangular unit formed by $90^{\circ}$ bonds together with "compass" interactions that follow from Eq. (3). Such a structure is common for a number of oxides, e.g., layered compounds $\mathrm{ABO}_{2}$ (where A and $\mathrm{B}$ are alkali and TM ions, respectively). The triangular lattice of magnetic ions in an $\mathrm{ABO}_{2}$ structure can be depleted down to a honeycomb lattice (by periodic replacements of TM ions with non-magnetic ones). One then obtains an $\mathrm{A}_{2} \mathrm{BO}_{3}$ compound, which has a hexagonal unit shown in Fig. 3 (b). There are three nonequivalent bonds, each being perpendicular to one of the cubic axes $x, y, z$. Then, according to Eq. (3) the spin coupling, e.g., on a $(x)$-bond is of $S_{i}^{x} S_{j}^{x}$ type, precisely as in the Kitaev model. The honeycomb lattice provides a particularly striking example of new physics introduced by strong SO coupling: the Heisenberg model is converted into the Kitaev model with a spin-liquid ground state.

The compound $\mathrm{Li}_{2} \mathrm{RuO}_{3}$ [28] represents a physical example of the $\mathrm{A}_{2} \mathrm{BO}_{3}$ structure. By replacement of spinone $\mathrm{Ru}^{4+}$ with spin one-half $\operatorname{Ir}^{4+}$ ions, one may realize a strongly spin-orbit coupled Mott insulator with low energy physics described by the Kitaev model.

"Weak" ferromagnetism of $\mathrm{Sr}_{2} \mathrm{IrO}_{4}$.- As an example of a spin-orbit coupled Mott insulator, we discuss the layered compound $\mathrm{Sr}_{2} \mathrm{IrO}_{4}$, a $t_{2 g}$ analog of the undoped high- $T_{c}$ cuprate $\mathrm{La}_{2} \mathrm{CuO}_{4}$. In $\mathrm{Sr}_{2} \mathrm{IrO}_{4}$, a square lattice

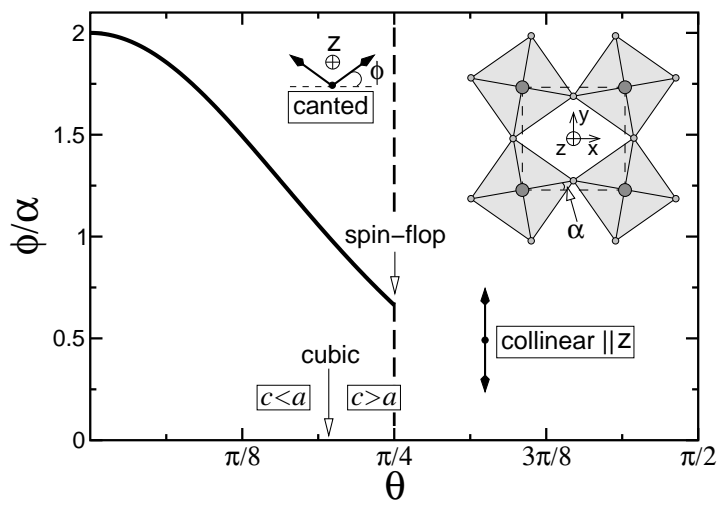

FIG. 4: The spin canting angle $\phi$ (in units of $\alpha$ ) as a function of the tetragonal distortion parameter $\theta$. Inset shows a sketch of an $\mathrm{IrO}_{2}$-plane. The oxygen octahedra are rotated by an angle $\pm \alpha$ about $z$-axis forming a two sublattice structure. In the cubic case, $\theta \simeq \pi / 5$, one has $\phi=\alpha$ exactly. The spin-flop transition from the in-plane canted spin state to a collinear Néel ordering along $z$-axis occurs at $\theta=\pi / 4$.

of $\mathrm{Ir}^{4+}$ ions is formed by corner-sharing $\mathrm{IrO}_{6}$ octahedra, elongated along the $c$-axis and rotated about it by $\alpha \simeq 11^{\circ}[19$. (see Fig. 44). The compound undergoes a magnetic transition at $\sim 240 \mathrm{~K}$ displaying a weak FM, which can be ascribed to a Dzyaloshinsky-Moriya (DM) interaction. The puzzling fact is that "weak" FM moment is in fact unusually large, $M_{\mathrm{FM}} \simeq 0.14 \mu_{B}[20]$ which is two-orders of magnitude larger than that in $\mathrm{La}_{2} \mathrm{CuO}_{4}$ [29]. A simple estimate gives a spin canting angle $\phi \simeq 8^{\circ}$ which is close to $\alpha$, i.e., the ordered spins seem to rigidly follow the staggered rotations of octahedra. Here we show that the strong $\mathrm{SO}$ coupling scenario gives a natural explanation of this observation.

We first show the dominant part of the Hamiltonian for $\mathrm{Sr}_{2} \mathrm{IrO}_{4}$ neglecting the Hund's coupling for a moment. Accounting for the rotations of $\mathrm{IrO}_{6}$ octahedra, we find:

$$
\mathcal{H}=J \vec{S}_{i} \cdot \vec{S}_{j}+J_{z} S_{i}^{z} S_{j}^{z}+\vec{D} \cdot\left[\vec{S}_{i} \times \vec{S}_{j}\right] .
$$

Here, the isotropic coupling $J=\nu_{1}\left(t_{s}^{2}-t_{a}^{2}\right)$, where $t_{s}=\sin ^{2} \theta+\frac{1}{2} \cos ^{2} \theta \cos 2 \alpha$, and $t_{a}=\frac{1}{2} \cos ^{2} \theta \sin 2 \alpha$. The second and third terms describe the symmetric and DM anisotropies, with $J_{z}=2 \nu_{1} t_{a}^{2}, \vec{D}=(0,0,-D)$, and $D=2 \nu_{1} t_{s} t_{a}$. [For $\alpha=0$, these terms vanish and we recover $J_{1}$-term of the $180^{\circ}$ result (2)]. As it follows from Eq. (4), the spin canting angle is given by a ratio $D / J \simeq 2 t_{a} / t_{s} \sim 2 \alpha$ which is independent of $\lambda$, and is solely determined by lattice distortions. This explains the large spin canting angle $\phi \sim \alpha$ in $\mathrm{Sr}_{2} \mathrm{IrO}_{4}$.

As in the case of weak SO coupling [30], the Hamiltonian (4) can in fact be mapped to the Heisenberg model $\overrightarrow{\tilde{S}}_{i} \cdot \overrightarrow{\tilde{S}}_{j}$ where operators $\overrightarrow{\tilde{S}}$ are obtained by a staggered rotation of $\vec{S}$ around the $z$-axis by an angle $\pm \phi$, with $\tan (2 \phi)=D / J$. Thus, at $J_{H}=0$, there is no true magnetic anisotropy. Once $J_{H}$-corrections are included, the 
Hamiltonian receives also the anisotropic terms:

$$
\tilde{\mathcal{H}}=\tilde{J}_{i} \cdot \overrightarrow{\widetilde{S}}_{j}-\Gamma_{1} \tilde{S}_{i}^{z} \tilde{S}_{j}^{z} \pm \Gamma_{2}\left(\tilde{S}_{i}^{x} \tilde{S}_{j}^{x}-\tilde{S}_{i}^{y} \tilde{S}_{j}^{y}\right)
$$

where $\tilde{J}=\nu_{1}\left(t_{s}^{2}+t_{a}^{2}\right)+\Gamma_{1}, \Gamma_{1}=\nu_{2} \cos ^{2} \theta \cos 2 \theta, \Gamma_{2}=$ $\nu_{2} \sin ^{2} \theta \cos ^{2} \theta$, and a \pm sign is taken for a bond along $x(y)$-axis. This Hamiltonian supports two types of spin orderings (see Fig. 4). For $\Gamma_{1}>0$ the spins form a canted structure in $x y$-plane. We find the out-of-plane magnon gap $\propto \sqrt{\tilde{J} \Gamma_{1}}$ of a classical origin, and much smaller in-plane gap $\propto \Gamma_{2}$ generated by quantum fluctuations. Shown in Fig. 4 is the spin canting angle $\phi$ (in units of $\alpha$ ) as a function of tetragonal distortion. In the cubic limit $\phi \equiv \alpha$, i.e., the spins simply rotate together with the oxygen octahedra. This suggests a strong magnetoelastic coupling in $\mathrm{Sr}_{2} \mathrm{IrO}_{4}$, and related phonon anomalies at the magnetic transition. The elongation $c>a$ (compression $c<a$ ) of octahedra leads to a decrease (increase) of $\phi$ and hence FM moment. At large $c / a$ ratio, $\Gamma_{1}$ changes sign. This marks a spin-flop transition to collinear order along the $z$-axis, which happens at $\theta=\pi / 4$, i.e., $\Delta=\lambda / 2$. This gives an upper estimate for the tetragonal splitting $\Delta<\lambda / 2 \simeq 190 \mathrm{meV}$ in $\mathrm{Sr}_{2} \mathrm{IrO}_{4}$, which agrees with optical data [21, 22]. Further, in the cubic limit we find $\Gamma_{1} / \tilde{J} \simeq 0.04$, which is much larger than that in $\mathrm{La}_{2} \mathrm{CuO}_{4}$, and far exceeds possible interlayer interactions [32]. This suggests that $X Y$-anisotropy is chiefly responsible for finite transition temperature in $\mathrm{Sr}_{2} \mathrm{IrO}_{4}$. From experimental value $T_{N}=240 \mathrm{~K}$ we estimate $\tilde{J} \simeq 45$ $\mathrm{meV}$ [33], which is a realistic value for a $t_{2 g}$-system [12].

We focused above on the Mott insulators [4], where the energy $\Delta_{p d}$ for a charge transfer from an oxygen to a TM ion is larger than $U$. Optical data show that the $p$ - $d$ transitions in $\mathrm{Sr}_{2} \mathrm{IrO}_{4}$ are indeed located at much higher energy than $d$ - $d$ ones [21, 22]. We, therefore, neglected the processes with two holes on the oxygen sites [31].

To conclude, we have considered magnetic interactions in Mott insulators with strong spin-orbit coupling. We find that the symmetry of low energy Hamiltonians is dictated by lattice geometry, opening a possibility to design exotic spin models like quantum compass and Kitaev models. Magnetic properties of the iridate $\mathrm{Sr}_{2} \mathrm{IrO}_{4}$ are explained. In general, spin-orbit coupled Mott insulators present an interesting new class of frustrated systems where the orbital, spin, and geometrical frustrations are superimposed via the spin-orbital entanglement, giving rise to unusual symmetries of interactions.

We would like to thank B. Keimer, D.I. Khomskii, and J. Chaloupka for stimulating discussions. G.J. acknowledges support by GNSF Grant No. 06-81-4-100.

* Also at E. Andronikashvili Institute of Physics, 0177 Tbilisi, Georgia
[1] M. Imada, A. Fujimori, and Y. Tokura, Rev. Mod. Phys. 70, 1039 (1998).

[2] Y. Tokura and N. Nagaosa, Science 288, 462 (2000).

[3] E. Dagotto, Science 309, 257 (2005).

[4] J. Zaanen, G. A. Sawatzky, and J. W. Allen, Phys. Rev. Lett. 55, 418 (1985).

[5] K.I. Kugel and D.I. Khomskii, Sov. Phys. Usp. 231, 25 (1982).

[6] H.F. Pen, J. van den Brink, D.I. Khomskii, and G.A. Sawatzky, Phys. Rev. Lett. 78, 1323 (1997).

[7] G. Jackeli and D.I. Khomskii, Phys. Rev. Lett. 100, 147203 (2008), and references therein.

[8] B. Keimer et al., Phys. Rev. Lett. 85, 3946 (2000).

[9] G. Khaliullin and S. Maekawa, Phys. Rev. Lett. 85, 3950 (2000); G. Khaliullin, Phys. Rev. B 64, 212405 (2001).

[10] J. Kanamori, Prog. Theor. Phys. 17, 177 (1956); 17, 197 (1956).

[11] G. Khaliullin, W. Koshibae, and S. Maekawa, Phys. Rev. Lett. 93, 176401 (2004).

[12] G. Khaliullin, Prog. Theor. Phys. Suppl. 160, 155 (2005).

[13] G. Chen and L. Balents, Phys. Rev. B 78, 094403 (2008).

[14] H. Katsura, N. Nagaosa, and A.V. Balatsky, Phys. Rev. Lett. 95, 057205 (2005).

[15] S. Murakami, N. Nagaosa, and S.-C. Zhang, Phys. Rev. Lett. 93, 156804 (2004).

[16] M.W. Haverkort et al., Phys. Rev. Lett. 101, 026406 (2008); G.-Q. Liu et al., ibid. 101, 026408 (2008).

[17] O.F. Schrimer et al., J. Phys. C 17, 1321 (1984).

[18] A. Kitaev, Ann. Phys. (N.Y.) 321, 2 (2006).

[19] Q. Huang et al., J. Solid State Chem. 112, 355 (1994).

[20] G. Cao et al., Phys. Rev. B 57, R11039 (1998).

[21] S.J. Moon et al., Phys. Rev. B 74, 113104 (2006).

[22] B.J. Kim et al., Phys. Rev. Lett. 101, 076402 (2008).

[23] A. Abragam and B. Bleaney, Electron Paramagnetic Resonance of Transition Ions (Clarendon Press, Oxford, 1970).

[24] $r_{1}=1 /(1-3 \eta), r_{2}=1 /(1-\eta)$ and $r_{3}=1 /(1+2 \eta)$.

[25] B. Douçot et al., Phys. Rev. B 71, 024505 (2005); J. Dorier, F. Becca, and F. Mila, ibid. 72, 024448 (2005).

[26] The Kitaev model [18] is defined on a honeycomb lattice with the following rule: label the three nonequivalent links of honeycomb lattice as $x, y$, and $z$, and couple on a given link only the corresponding Cartesian components of spin operators, i.e., $S_{i}^{x} S_{j}^{x}$ on $x$-links, etc.

[27] L.-M. Duan, E. Demler, and M.D. Lukin, Phys. Rev. Lett. 91, 090402 (2003); S. Trotzky et al., Science 319, 295 (2008); A. Micheli, G. K. Brennen, and P. Zoller, Nature Phys. 2, 341 (2006).

[28] Y. Miura et al., J. Phys. Soc. Jpn. 76, 033705 (2007).

[29] T. Thio et al., Phys. Rev. B 38, 905 (1988).

[30] L. Shekhtman, O. Entin-Wohlman, and A. Aharony, Phys. Rev. Lett. 69, 836 (1992).

[31] For completeness, we notice that in the case of a $180^{\circ}$ bond, the $p$ - $d$ charge transfer contributions do not change the Hamiltonian (2) and only lead to a trivial renormalization of the coupling constants. Conversely, on a $90^{\circ}-$ bond, such processes generate a term $J^{\prime}\left(\vec{S}_{i} \cdot \vec{S}_{j}-2 S_{i}^{\gamma} S_{j}^{\gamma}\right)$, composed of Heisenberg and compass models (see also Ref. 13). We find $J^{\prime}=\frac{1}{9} \frac{U_{p}}{\Delta_{p d}+U_{p} / 2} \frac{4 t^{2}}{\Delta_{p d}}$ which is small in the Mott insulator regime of $\Delta_{p d} \gg U_{p}, U$.

[32] B. Keimer et al., Phys. Rev. B 45, 7430 (1992).

[33] The estimate is based on $T_{N}$-equation derived by V.Yu. Irkhin and A.A. Katanin [Phys. Rev. B 60, 2990 (1999)]. 